\title{
Alterstice
}

Revue internationale de la recherche interculturelle

International Journal of Intercultural Research

Revista International de la Investigacion Intercultural

\section{Ceci n’est pas un éditorial}

\section{Yvan Leanza}

Volume 1, numéro 1, 2011

URI : https://id.erudit.org/iderudit/1077585ar

DOI : https://doi.org/10.7202/1077585ar

Aller au sommaire du numéro

Éditeur(s)

Alterstice

ISSN

1923-919X (numérique)

Découvrir la revue

Citer ce document

Leanza, Y. (2011). Ceci n'est pas un éditorial. Alterstice, 1(1), 1-2.

https://doi.org/10.7202/1077585ar d'utilisation que vous pouvez consulter en ligne.

https://apropos.erudit.org/fr/usagers/politique-dutilisation/ 


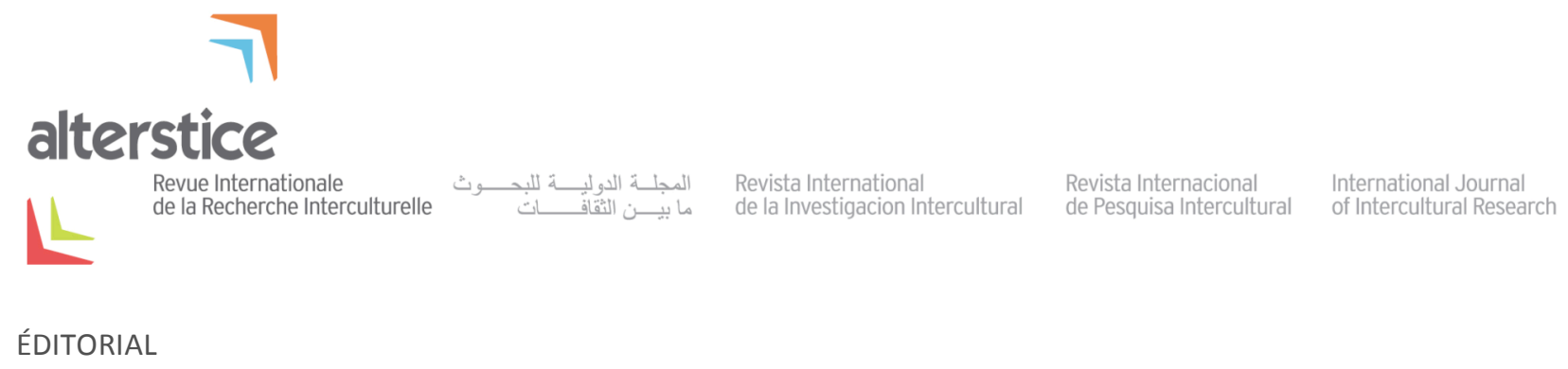

\title{
Ceci n'est pas un éditorial
}

\author{
Yvan Leanza $^{1}$
}

Ceci n'est pas un éditorial. C'est le résultat d'un long, même très long, processus de co-construction. Dans sa forme, il s'agit bien d'un éditorial, soit le mot du directeur de la revue pour présenter le numéro. Et certains diront probablement, avec raison, que toute production textuelle est le résultat d'un processus. Cependant, dans ce cas précis, celui que vous avez sous les yeux, le processus a impliqué de nombreuses personnes et s'est étendu sur plus de 25 ans.

II a d'abord fallu créer l'Association pour la Recherche InterCulturelle (ARIC), ce qui a eu lieu en 1984. Parmi les fondateurs, l'idée d'avoir une revue scientifique était déjà présente, et quelques-unes de ces personnes participent au comité scientifique d'Alterstice ou à ce premier numéro, voire aux deux comme Margalit Cohen-Emerique. L'ARIC a bien eu son bulletin, qui est progressivement passé d'une feuille d'information entre les membres avec un ou deux articles scientifiques à une proto-revue avec comité de lecture, en particulier sous la responsabilité de Colette Sabatier (Université de Bordeaux, France), puis de Tania Ogay (Université de Fribourg, Suisse), Luc Collès (Université de Louvain, Belgique) et moi-même (Université Laval, Canada). Cependant, à l'heure des nouvelles technologies de l'information, et des exigences élevées des institutions universitaires et de celles finançant la recherche, il n'était plus efficace de garder ce format hybride du point de vue du contenu comme de la forme.

Bien que I'ARIC compte de nombreux et brillants membres répartis sur quatre continents, un tel projet ne pouvait voir le jour sans un ancrage localisé là où il y a une expertise en matière de publication scientifique (et des ressources). C'est ainsi que l'équipe de recherche Migration et EThnicité dans les Interventions en Santé et en Services sociaux (METISS) de Montréal, sous la direction de Catherine Montgomery (Université McGill, Canada), a accepté de se joindre au projet dès 2008. METISS avait également sa propre série de publications, les Cahiers METISS, là aussi hybride par son contenu (à la fois orienté vers la pratique et la recherche) et disponible en ligne, mais souhaitait distinguer les publications à destination des praticiens de celles à destination des chercheurs. C'est par l'appui moral et financier de METISS que le projet a pu prendre forme. L'équipe METISS compte parmi ses membres Jacques Rhéaume (Université du Québec à Montréal, Canada) qui est directeur de la revue Nouvelles Pratiques Sociales et qui a accepté de se joindre au premier comité de direction de la revue.

La constitution d'une revue a été officialisée par le Conseil de l'ARIC lors du colloque de Messina en septembre 2008, peu après que METISS ait donné son approbation à ce projet ambitieux. Plus tard, le Centre interuniversitaire d’Études sur les Langues, les Arts et les Traditions (CÉLAT) sous la direction de Francine Saillant (Université Laval, Canada) est venu prêter main-forte au projet, non seulement par l'expérience de Francine Saillant elle-même, qui a été longtemps directrice de la revue Anthropologie et Société, mais aussi par des aides financières ponctuelles qui permettent d'effectuer un travail professionnel dans la révision et la mise en page des textes. Sans oublier, bien entendu, le rayonnement de la revue dans un autre réseau multidisciplinaire dont la programmation scientifique 2011-2017 porte sur « le vivre-ensemble à l'épreuve de la pluralisation croissante des sociétés ».

Je tiens à rappeler ici quelques principes de fonctionnement de la revue, principes qui ont été discutés et approuvés par un comité ad hoc réunissant des membres du Conseil de l'ARIC et de METISS. Ce comité a établi la structure de la revue avant son existence matérielle et a été dissous peu avant la création de la revue, soit en 
avril 2010. Les instances de la revue sont le comité de direction composé de cinq membres, dont le directeur (ou la directrice) et le comité scientifique. Le comité de direction veille au fonctionnement quotidien de la revue. Il est composé de représentants des trois réseaux porteurs du projet. Le comité scientifique veille à l'orientation de la revue, par exemple en suggérant des thèmes pour les numéros à venir et en arbitrant en cas d'évaluations contradictoires d'un article soumis. Il veille également à la diffusion de la revue dans différents milieux universitaires et professionnels. La composition du comité scientifique reflète à la fois la multidisciplinarité de la recherche interculturelle et la diversité socioculturelle et géographique des chercheurs associés aux trois réseaux. Un équilibre qu'il n'a pas été facile à trouver, mais qu'il est nécessaire de maintenir pour l'avenir.

D’un point de vue pratique, voici les principes directeurs de la revue:

- Les numéros d'Alterstice sont thématiques et font appel à des éditeurs invités. II est aussi possible de soumettre des articles hors thème et des notes de lecture (compte rendu d'ouvrage).

- Bien que la langue " première " d'Alterstice, comme celle des trois réseaux auxquels elle est associée, soit le français, il est possible de soumettre des articles en arabe, espagnol, portugais et anglais.

- Après une évaluation sommaire par les éditeurs invités et le comité de direction, les articles sont soumis en aveugle à deux évaluateurs externes. L'auteur doit répondre aux commentaires qui lui sont faits et modifier son texte si nécessaire avant la publication.

Je ne pourrais terminer ce premier éditorial sans remercier les personnes qui ont cru dans le projet et qui m'ont soutenu pour le mettre en forme, en particulier les membres du comité ad hoc: Émile-Henry Riard (Université de Picardie Jules Verne, France), Fabienne Rio (Unité de recherche Migration et Société, Université de Nice et Université de Paris 8, France), Fatima Moussa (Université d'Alger, Algérie), Ghazi Chakroun (Université de Sfax, Tunisie), Lucienne Borges Matins (Université de Santa-Catarina, Brésil), Michèle Vatz Laaroussi (Université de Sherbrooke, Canada), Reinaldo Fleuri (Université de Santa Catarina, Brésil), René Mokounkolo (Université de Tours, France), Catherine Montgomery (Université McGill, Canada) et Jacques Rhéaume (Université du Québec à Montréal, Canada). Je remercie également les membres du comité scientifique pour leur engagement dans ce projet un peu fou, ainsi que mes collègues du comité de direction pour leur écoute, leur disponibilité et leurs sages conseils. Je tiens à souligner la contribution d’Anna Olivier, réviseure, qui a mis généreusement son expertise au service de ce premier numéro. Et pour finir, mes remerciements vont à Anahy Gajardo, qui a accepté de co-éditer ce numéro, une entreprise risquée qui devrait établir un jalon pour Alterstice.

En ces temps d'indignation légitime, parfois contrée violemment, je fais le souhait que cette revue s'applique à présenter les connaissances nouvelles (ou un peu moins), celles qui se situent dans l'alterstice - un entre-deux qui transforme - et qui permettront non seulement l'avancée des sciences sociales et humaines, mais aussi le développement de sociétés plurielles plus justes.

Bonne lecture!

\author{
Rattachement de l'auteur \\ ${ }^{1}$ Université Laval, Québec, Canada
}

Correspondance

alterstice@gmail.com

Pour citer cet article :

Leanza, Y. (2011). Ceci n'est pas un éditorial. Alterstice, 1(1), 1-2. 